\title{
LEVY'S PHENOMENON FOR ENTIRE FUNCTIONS OF SEVERAL VARIABLES
}

\author{
A.O. KURYLIAK, O.B. SKASKIV, O.V. ZRUM
}

\begin{abstract}
For entire functions $f(z)=\sum_{n=0}^{+\infty} a_{n} z^{n}, z \in \mathbb{C}$, P. Lévy (1929) established that in the classical Wiman's inequality $M_{f}(r) \leqslant \mu_{f}(r)\left(\ln \mu_{f}(r)\right)^{1 / 2+\varepsilon}, \varepsilon>0$, which holds outside a set of finite logarithmic measure, the constant $1 / 2$ can be replaced almost surely in some sense by $1 / 4$; here $M_{f}(r)=\max \{|f(z)|:|z|=r\}, \mu_{f}(r)=\max \left\{\left|a_{n}\right| r^{n}: n \geqslant 0\right\}, r>$ 0 . In this paper we prove that the phenomenon discovered by P. Lévy holds also in the case of Wiman's inequality for entire functions of several variables, which gives an affirmative answer to the question of A. A. Goldberg and M. M. Sheremeta (1996) on the possibility of this phenomenon.
\end{abstract}

Keywords: Levy's phenomenon, random entire functions of several variables, Wiman's inequality

Mathematics Subject Classification: 30B20, 30D20

\section{INTRODUCTION}

For an entire function of the form

$$
f(z)=\sum_{n=0}^{+\infty} a_{n} z^{n}
$$

we denote $M_{f}(r)=\max \{|f(z)|:|z|=r\}, \mu_{f}(r)=\max \left\{\left|a_{n}\right| r^{n}: n \geqslant 0\right\}, r>0$. It is well known ([1], [2]) that for each nonconstant entire function $f$ and each $\varepsilon>0$ the following inequality

$$
M_{f}(r) \leqslant \mu_{f}(r)\left(\ln \mu_{f}(r)\right)^{1 / 2+\varepsilon}
$$

holds for $r>1$ outside an exceptional set $E_{f}(\varepsilon)$ of finite logarithmic measure $\left(\int_{E_{f}(\varepsilon)} \frac{d r}{r}<+\infty\right)$.

In this paper we consider entire functions of $p$ complex variables

$$
f(z)=f\left(z_{1}, \ldots, z_{p}\right)=\sum_{\|n\|=0}^{+\infty} a_{n} z^{n},
$$

where $z^{n}=z_{1}^{n_{1}} \ldots z_{p}^{n_{p}}, p \in \mathbb{N}, n=\left(n_{1}, \ldots, n_{p}\right) \in \mathbb{Z}_{+}^{p},\|n\|=\sum_{j=1}^{p} n_{j}$. For $r=\left(r_{1}, \ldots, r_{p}\right) \in$ $\mathbb{R}_{+}^{p}$ we denote

$$
\begin{aligned}
B(R)= & \left\{t \in \mathbb{R}_{+}^{p}: t_{j} \geqslant R_{j}, j \in\{1, \ldots, p\}\right\}, R=\left(R_{1}, \ldots, R_{p}\right), \ln _{2} x=\ln \ln x, \\
& r^{\wedge}=\min _{1 \leqslant i \leqslant p} r_{i}, M_{f}(r)=\max \left\{|f(z)|:\left|z_{1}\right|=r_{1}, \ldots,\left|z_{p}\right|=r_{p}\right\}, \\
& \mu_{f}(r)=\max \left\{\left|a_{n}\right| r_{1}^{n_{1}} \ldots r_{p}^{n_{p}}: n \in \mathbb{Z}_{+}^{p}\right\}, \mathfrak{M}_{f}(r)=\sum_{\|n\|=0}^{+\infty}\left|a_{n}\right| r^{n} .
\end{aligned}
$$

A.O. Kuryliak, O.B. Skaskiv, O.V. Zrum Levy's Phenomenon for entire functions of Several VARIABLES.

(c) A.O. KurYliak, O.B. Skaskiv, O.V. Zrum 2014.

Submitted October 7, 2014. 
By $\Lambda^{p}$ we denote the class of entire functions such that $\frac{\partial}{\partial z_{j}} f(z) \not \equiv 0$ in $\mathbb{C}^{p}$ for each $j \in$ $\{1, \ldots, p\}$. We say that a subset $E$ of $\mathbb{R}_{+}^{p}$ is a set of asymptotically finite logarithmic measure [9] if $E$ is Lebesgue measurable in $\mathbb{R}_{+}^{p}$ and there exists an $R \in \mathbb{R}_{+}^{p}$ such that $E \cap B(R)$ is a set of finite logarithmic measure, i.e.

$$
\int_{E \cap B(R)} \cdots \int_{j=1}^{p} \frac{d r_{j}}{r_{j}}<+\infty
$$

For entire functions (2) analogues of inequality (1) were proved in [3, 5, 6, 9]. Also analogues of inequality (1) without exceptional sets for entire functions of several complex variables can be found in [10].

In particular, the following statement was proved in [9].

Theorem 1. Let $f \in \Lambda^{p}$ and $\delta>0$.

a) Then there exist $R \in \mathbb{R}_{+}^{p}$ and a subset $E$ of $B(R)$ of finite logarithmic measure such that for each $r \in B(R) \backslash E$ we have

$$
\mathfrak{M}_{f}(r) \leqslant \mu_{f}(r)\left(\prod_{i=1}^{p} \ln ^{p-1} r_{i} \cdot \ln ^{p} \mu_{f}(r)\right)^{1 / 2+\delta} .
$$

b) If for some $\alpha \in \mathbb{R}_{+}^{p}$ we have $\mathfrak{M}(r) \geqslant \exp \left(r^{\alpha}\right)=\exp \left(r_{1}^{\alpha_{1}} \ldots r_{p}^{\alpha_{p}}\right)$, as $r^{\wedge} \rightarrow+\infty$ or more generally, for each $\beta>0$

$$
\int \ldots \int \frac{\prod_{i=1}^{p} d r_{i}}{r_{1} r_{2} \ldots r_{p} \ln ^{\beta} \mathfrak{M}_{f}(r)}<+\infty, \text { as } S^{\wedge} \rightarrow+\infty,
$$

then there exist $R \in \mathbb{R}_{+}^{p}$ and a subset $E$ of $B(R)$ of finite logarithmic measure such that for each $r \in B(R) \backslash E$ we have

$$
\mathfrak{M}_{f}(r) \leqslant \mu_{f}(r) \ln ^{p / 2+\delta} \mu_{f}(r) .
$$

\section{WIMAN'S TYPE INEQUALITY}

FOR RANDOM ENTIRE FUNCTIONS OF SEVERAL VARIABLES

Let $\Omega=[0,1]$ and $P$ be the Lebesgue measure on $\mathbb{R}$. We consider the Steinhaus probability space $(\Omega, \mathcal{A}, P)$, where $\mathcal{A}$ is the $\sigma$-algebra of Lebesgue measurable subsets of $\Omega$. Let $X=\left(X_{n}(t)\right)$ be some sequence of random variables defined in this space. For an entire function of the form $f(z)=\sum_{n=0}^{+\infty} a_{n} z^{n}$ by $K(f, X)$ we denote the class of random entire functions

$$
f(z, t)=\sum_{n=0}^{+\infty} a_{n} X_{n}(t) z^{n} .
$$

In what follows, the notion "almost surely" will be employed in the sense that the corresponding property holds almost everywhere with respect to Lebesgue measure $P$ on $\Omega=[0,1]$. We say that some relation holds almost surely in the class $K(f, X)$ if it holds for each entire function $f(z, t)$ of the form (5) almost surely in $t$.

In the case $\mathcal{R}=\left(X_{n}(t)\right)$ is the Rademacher sequence, i.e. $\left(X_{n}(t)\right)$ is a sequence of independent uniformly distributed random variables on $[0,1]$ such that $P\left\{t: X_{n}(t)= \pm 1\right\}=1 / 2$, P. Levy [7] proved that for each entire function we can replace the constant $1 / 2$ by $1 / 4$ in the inequality (1) almost surely in the class $K(f, \mathcal{R})$. Later P. Erdös and A. Rényi [8] proved the same result for the class $K(f, H)$, where $H=\left(e^{2 \pi i \omega_{n}(t)}\right)$ is the Steinhaus sequence, i.e. $\left(\omega_{n}(t)\right)$ is a sequence of independent uniformly distributed random variables on $[0,1]$. This statement is true also for 
each class $K(f, X)$, where $X=\left(X_{n}(t)\right)$ is multiplicative system (MS) uniformly bounded by the number 1 . That is for each $n \in \mathbb{N}$ and $t \in[0,1]$ we have $\left|X_{n}(t)\right| \leqslant 1$ and

$$
\left(\forall 1 \leqslant i_{1}<i_{2}<\cdots<i_{k}\right): \mathbf{M}\left(X_{i_{1}} X_{i_{2}} \cdots X_{i_{k}}\right)=0,
$$

where $\mathbf{M} \xi$ is the expected value of a random variable $\xi([15]-[16])$.

In the spring of 1996 during the report of P. V. Filevych at the Lviv seminar of the theory of analytic functions, professors A. A. Goldberg and M. M. Sheremeta posed the following question (see [12]): Does Levy's effect take place for analogues of Wiman's inequality for entire functions of several complex variables?

In the papers [12]-[14] we have found an affirmative answer to this question for Fenton's inequality [4] for entire functions of two complex variables.

In this paper we will give answer to this question for Wiman's type inequality in [9] for entire functions of several complex variables.

The exceptional set in our statements is "smaller" than the exceptional set in the corresponding theorems from [4, [12]-[14]. The method of proof in this paper differs from the method of the papers [4], [12]-[14].

Let $Z=\left(Z_{n}(t)\right)$ be a complex sequence of random variables $Z_{n}(t)=X_{n}(t)+i Y_{n}(t)$ such that both $X=\left(X_{n}(t)\right)$ and $Y=\left(Y_{n}(t)\right)$ are real MS and $K(f, Z)$ the class of random entire functions of the form

$$
f(z, t)=\sum_{\|n\|=0}^{+\infty} a_{n} Z_{n}(t) z_{1}^{n_{1}} \ldots z_{p}^{n_{p}} .
$$

Theorem 2. Let $Z=\left(Z_{n}(t)\right)$ be a $M S$ uniformly bounded by the number $1, \delta>0, f \in \Lambda^{p}$.

a) Then almost surely in $K(f, Z)$ there exist $R \in \mathbb{R}_{+}^{p}$ and a subset $E^{*}$ of $B(R)$ of finite logarithmic measure such that for each $r \in B(R) \backslash E^{*}$ we have

$$
M_{f}(r, t)=\max _{|z|=r}|f(z, t)| \leqslant \mu_{f}(r)\left(\ln ^{p} \mu_{f}(r) \cdot \prod_{i=1}^{p} \ln ^{p-1} r_{i}\right)^{1 / 4+\delta} .
$$

b) If for some $\alpha \in \mathbb{R}_{+}^{p}$ we have

$$
\mathfrak{M}(r) \geqslant \exp \left(r^{\alpha}\right)=\exp \left(r_{1}^{\alpha_{1}} \ldots r_{p}^{\alpha_{p}}\right) \text { as } r^{\wedge} \rightarrow+\infty
$$

or more generally, for each $\beta>0$ inequality (4) holds, then almost surely in $K(f, Z)$ there exist $R \in \mathbb{R}_{+}^{p}$ and a subset $E$ of $B(R)$ of finite logarithmic measure such that for each $r \in B(R) \backslash E$ we get

$$
M_{f}(r, t) \leqslant \mu_{f}(r) \ln ^{p / 4+\delta} \mu_{f}(r) .
$$

Lemma 1 ([10]). Let $X=\left(X_{n}(t)\right)$ be a MS uniformly bounded by the number 1 . Then for each $\beta>0$ there exists a constant $A_{\beta p}>0$, which depends on $p$ and $\beta$ only such that for each $N \geqslant N_{1}(p)=\max \{p, 4 \pi\}$ and $\left\{c_{n}:\|n\| \leqslant N\right\} \subset \mathbb{C}$ we have

$$
P\left\{t: \max \left\{\left|\sum_{\|n\|=0}^{N} c_{n} X_{n}(t) e^{i n_{1} \psi_{1}} \ldots e^{i n_{p} \psi_{p}}\right|: \psi \in[0,2 \pi]^{p}\right\} \geqslant A_{\beta p} S_{N} \ln ^{\frac{1}{2}} N\right\} \leqslant \frac{1}{N^{\beta}},
$$

where $S_{N}^{2}=\sum_{\|n\|=0}^{N}\left|c_{n}\right|^{2}$.

By $H$ we denote the class of function $h: \mathbb{R}_{+}^{p} \rightarrow \mathbb{R}_{+}$such that

$$
\int_{1}^{+\infty} \ldots \int_{1}^{+\infty} \frac{d u_{1} \ldots d u_{p}}{h(u)}<+\infty
$$


For each $i \in\{1, \ldots, p\}$ we also define

$$
\partial_{i} \ln \mathfrak{M}_{f}(r)=r_{i} \frac{\partial}{\partial r_{i}}\left(\ln \mathfrak{M}_{f}(r)\right)=\frac{1}{\mathfrak{M}_{f}(r)} \sum_{\|n\|=0}^{+\infty} n_{i}\left|a_{n}\right| r^{n}
$$

Lemma $2([9])$. Let $h \in H$. Then there exist $R \in \mathbb{R}_{+}^{p}$ and a subset $E^{\prime}$ of $B(R)$ of finite logarithmic measure such that for each $r \in B(R) \backslash E^{\prime}$ and $s \in\{1, \ldots, p\}$ we have

$$
\partial_{s} \ln \mathfrak{M}_{f}(r) \leqslant h\left(\ln r_{1}, \ldots, \ln r_{s-1}, \ln \mathfrak{M}_{f}(r), \ln r_{s+1}, \ldots, \ln r_{p}\right) .
$$

Proof of Theorem 2. Without loss of generality we may suppose that $Z=X=\left(X_{n}(t)\right)$ is a MS. Indeed, if $Z_{n}(t)=X_{n}(t)+i Y_{n}(t)$, we obtain

$$
f(z, t)=\sum_{\|n\|=0}^{+\infty} a_{n} X_{n}(t) z^{n}+\sum_{\|n\|=0}^{+\infty} i a_{n} Y_{n}(t) z^{n}=f_{1}(z, t)+f_{2}(z, t),
$$

where $f_{1}, f_{2} \in K(f, X)$, and

$$
\max \left\{\mu\left(r, f_{1}(\cdot, t)\right), \mu\left(r, f_{2}(\cdot, t)\right)\right\} \leqslant \mu(r, f)=\max \left\{\left|a_{n}\right| r_{1}^{n_{1}} \ldots r_{p}^{n_{p}}: n \in \mathbb{Z}_{+}^{p}\right\}
$$

for each $r \in \mathbb{R}_{+}^{p}$ and $t \in[0,1]$. Hence, by inequality (6) we obtain that there exists a set $E_{0}$ of asymptotically finite logarithmic measure such that for each $r \in B(R) \backslash E_{0}$ almost surely in $K(f, Z)$

$$
M_{f_{j}}(r, t) \leqslant \mu_{f}(r)\left(\prod_{i=1}^{p} \ln ^{p-1} r_{i} \cdot \ln ^{p} \mu_{f}(r)\right)^{1 / 4+\delta_{0}}, \quad j \in\{1,2\}, \delta_{0}>0 .
$$

Thus, for $R^{\wedge}$ great enough and for each $r \in B(R) \backslash E_{0}$ almost surely in $K(f, Z)$ we get

$$
\begin{aligned}
M_{f}(r, t) & \leqslant M_{f_{1}}(r, t)+M_{f_{2}}(r, t) \\
& \leqslant 2 \mu_{f}(r)\left(\prod_{i=1}^{p} \ln ^{p-1} r_{i} \cdot \ln ^{p} \mu_{f}(r)\right)^{1 / 4+\delta_{0}}<\mu_{f}(r)\left(\prod_{i=1}^{p} \ln ^{p-1} r_{i} \cdot \ln ^{p} \mu_{f}(r)\right)^{1 / 4+2 \delta_{0}} .
\end{aligned}
$$

For each $j \in\{1, \ldots, p\}$ we have

$$
\lim _{r_{j} \rightarrow+\infty} \mu_{f}\left(r_{1}^{0}, \ldots, r_{j-1}^{0}, r_{j}, r_{j+1}^{0}, \ldots, r_{p}^{0}\right)=+\infty
$$

for fixed $r_{i}^{0}>0, i \in\{1, \ldots, p\} \backslash\{j\}$. Indeed, if $10 p$ does not hold, there exists a constant $C>0$ such that for each $r_{j}>r_{j}^{*}$ we have $\mu_{f}\left(r_{1}^{0}, \ldots, r_{j-1}^{0}, r_{j}, r_{j+1}^{0}, \ldots, r_{p}^{0}\right)<C<+\infty$. Hence, $\#\left\{n_{j} \geqslant 1: a_{n} \neq 0\right\}=0$ and $\frac{\partial}{\partial z_{j}} f(z) \equiv 0$ in $\mathbb{C}^{p}$. So, $f \notin \Lambda^{p}$, which gives a contradiction.

For $k \in \mathbb{N} \cup\{0\}$ we denote $G_{k}=\left\{r=\left(r_{1}, \ldots, r_{p}\right) \in \mathbb{R}_{+}^{p}: k \leqslant \ln \mu_{f}(r)<k+1\right\} \cap[1 ;+\infty)^{p}$. Then $G_{k} \neq \varnothing$ for $k \geqslant k_{0}$ and by $(10)$ we obtain that for each $k$ the set $G_{k}$ is a bounded set. Let $G_{k}^{+}=\bigcup_{j=k}^{+\infty} G_{j}$ and

$$
h(r)=\prod_{i=1}^{p} r_{i} \ln ^{1+\delta_{1}} r_{i} \in H, \delta_{1}>0 .
$$

By Lemma 2 there exist $R_{j} \in \mathbb{R}_{+}^{p}$ and a subset $E_{j}$ of $B\left(R_{j}\right)$ of finite logarithmic measure such that for each $r \in B\left(R_{j}\right) \backslash E_{j}$ and $j \in\{1, \ldots, p\}$ we have

$$
\begin{aligned}
\sum_{\|n\|=0}^{+\infty} n_{i}\left|a_{n}\right| r^{n} & \leqslant \mathfrak{M}_{f}(r) h\left(\ln r_{1}, \ldots, \ln r_{s-1}, \ln \mathfrak{M}_{f}(r), \ln r_{s+1}, \ldots, \ln r_{n}\right) \\
& \leqslant \mathfrak{M}_{f}(r) \ln \mathfrak{M}_{f}(r) \ln _{2}^{1+\delta_{1}} \mathfrak{M}_{f}(r) \prod_{i=1, i \neq j}^{p} \ln r_{i} \ln _{2}^{1+\delta_{1}} r_{i} .
\end{aligned}
$$

We can choose $R \in \mathbb{R}_{+}^{p}$ so that $B(R) \subset\left(\bigcap_{j=1}^{p} B\left(R_{j}\right)\right) \cap\left[e^{e^{2}},+\infty\right)^{p}$. 
Thus, for $R^{\wedge}$ great enough and for each $r \in B(R) \backslash\left(\cup_{i=1}^{p} E_{i}\right)$ we obtain

$$
\begin{aligned}
\sum_{\|n\|=0}^{+\infty}\|n\|\left|a_{n}\right| r^{n} & \leqslant \mathfrak{M}_{f}(r) \ln \mathfrak{M}_{f}(r) \ln _{2}^{1+\delta_{1}} \mathfrak{M}_{f}(r) \sum_{j=1}^{p}\left(\prod_{i=1, i \neq j}^{p} \ln r_{i} \ln _{2}^{1+\delta_{1}} r_{i}\right) \\
& \leqslant p \cdot \mathfrak{M}_{f}(r) \ln ^{1+\delta_{1} / 2} \mathfrak{M}_{f}(r) \prod_{i=1}^{p} \ln r_{i} \ln _{2}^{1+\delta_{1}} r_{i},
\end{aligned}
$$

By Theorem 1, we get that for $R^{\wedge}$ great enough and for each $r \in B(R) \backslash\left(\bigcup_{i=1}^{p} E_{i}\right)$

$$
\begin{aligned}
\sum_{\|n\|=0}^{+\infty}\|n\|\left|a_{n}\right| r^{n} \leqslant & p \mu_{f}(r)\left(\prod_{i=1}^{p} \ln ^{p-1} r_{i} \cdot \ln ^{p} \mu_{f}(r)\right)^{1 / 2+\delta_{1}} \times \\
& \times\left(\ln \mu_{f}(r)+\left(\frac{1}{2}+\delta_{1}\right)\left((p-1) \sum_{i=1}^{p} \ln _{2} r_{i}+p \ln _{2} \mu_{f}(r)\right)\right)^{1+\delta_{1} / 2} \prod_{i=1}^{p} \ln r_{i} \ln _{2}^{1+\delta_{1}} r_{i} \\
\leqslant & \mu_{f}(r)\left(\ln \mu_{f}(r)\right)^{p / 2+(p+1) \delta_{1}+1}\left(\prod_{i=1}^{p} \ln r_{i}\right)^{(p-1)\left(1 / 2+\delta_{1}\right)+1}\left(\prod_{i=1}^{p} \ln _{2} r_{i}\right)^{2+3 \delta_{1} / 2}
\end{aligned}
$$

since $a_{1} x_{1}+\cdots+a_{k} x_{k}<x_{1} \cdot \ldots \cdot x_{k}$ for $x^{\wedge} \geqslant 1$ great enough, $x=\left(x_{1}, \ldots, x_{k}\right)$. Therefore, as $\delta_{2}=(p+1) \delta_{1}$, for $R^{\wedge}$ great enough and for each $r \in B(R) \backslash\left(\bigcup_{i=1}^{p} E_{i}\right)$ we obtain

$$
\sum_{\|n\|=0}^{+\infty}\|n\|\left|a_{n}\right| r^{n} \leqslant \mu_{f}(r) \ln ^{p / 2+1+\delta_{2}} \mu_{f}(r) \prod_{i=1}^{p}\left(\ln ^{p} r_{i} \ln _{2}^{2} r_{i}\right)^{1+\delta_{2}}
$$

Hence,

$$
\begin{aligned}
\sum_{\|n\| \geqslant d}\left|a_{n}\right| r^{n} & \leqslant \sum_{\|n\| \geqslant d} \frac{\|n\|}{d}\left|a_{n}\right| r^{n}=\frac{1}{d} \sum_{\|n\| \geqslant d}\|n\|\left|a_{n}\right| r^{n} \\
& \leqslant \frac{1}{d} \mu_{f}(r) \ln ^{p / 2+1+\delta_{2}} \mu_{f}(r) \prod_{i=1}^{p}\left(\ln ^{p} r_{i} \ln _{2}^{2} r_{i}\right)^{1+\delta_{2}}=\mu_{f}(r)
\end{aligned}
$$

where

$$
d=d(r)=\ln ^{p / 2+1+\delta_{2}} \mu_{f}(r) \prod_{i=1}^{p}\left(\ln ^{p} r_{i} \ln _{2}^{2} r_{i}\right)^{1+\delta_{2}}
$$

Let $G_{k}^{*}=G_{k} \backslash E_{p+1}, E_{p+1}=\bigcup_{i=1}^{p}\left(E_{i} \cup E^{*}\right) \cup\left(\bigcup_{i=1}^{k_{0}-1} G_{i}\right)$. By $I$ we denote the set of integers $k \geqslant k_{0}$ such that $G_{k}^{*} \neq \varnothing$. Then $\# I=+\infty$. For $k \in I$ we choose a sequence $r^{(k)} \in G_{k}^{*}$. Then for each $r \in G_{k}^{*}$ we get

$$
\mu_{f}\left(r^{(k)}\right)<e^{k+1} \leqslant e \mu_{f}(r), \quad \mu_{f}(r)<e^{k+1}<e \mu_{f}\left(r^{(k)}\right),
$$

and also

$$
\bigcup_{k \in I} G_{k}^{*}=\bigcup_{k \in I} G_{k} \backslash E_{p+1}=\bigcup_{k=1}^{+\infty} G_{k} \backslash E_{p+1}=[1 ;+\infty)^{p} \backslash E_{p+1} .
$$

For $k \in I$ we denote $N_{k}=\left[2 d_{1}\left(r^{(k)}\right)\right]$, where

$$
d_{1}(r)=\ln ^{p / 2+1+\delta_{2}}\left(e \mu_{f}(r)\right) \prod_{i=1}^{p}\left(\ln ^{p} r_{i} \ln _{2}^{2} r_{i}\right)^{1+\delta_{2}}
$$


and for $r \in G_{k}^{*}$

$$
W_{N_{k}}(r, t)=\max \left\{\left|\sum_{\|n\| \leqslant N_{k}} a_{n} r_{1}^{n_{1}} \ldots r_{p}^{n_{p}} e^{i n_{1} \psi_{1}+\ldots+i n_{p} \psi_{p}} X_{n}(t)\right|: \psi \in[0,2 \pi]^{p}\right\} .
$$

For a Lebesgue measurable set $G \subset G_{k}^{*}$ and for $k \in I$ we denote

$$
\nu_{k}(G)=\frac{\operatorname{meas}_{p}(G)}{\operatorname{meas}_{p}\left(G_{k}^{*}\right)},
$$

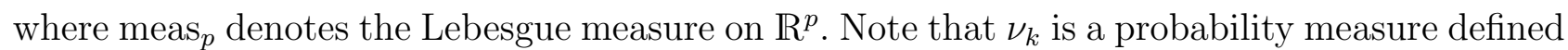
on the family of Lebesgue measurable subsets of $G_{k}^{*}$.

Let $\Omega=\bigcup_{k \in I} G_{k}^{*}$ and $I=\left\{k_{j}: j \geqslant 0\right\}$, where $k_{j}<k_{j+1}, j \geqslant 0$. Without loss of generality we may assume that $k_{0}=0$. Then $E_{p+1}=\bigcup_{i=1}^{p}\left(E_{i} \cup E^{*}\right)$. For Lebesgue measurable subsets $G$ of $\Omega$ we denote

$$
\nu(G)=\sum_{j=0}^{+\infty} \frac{1}{2^{k_{j}}}\left(1-\left(\frac{1}{2}\right)^{k_{j+1}-k_{j}}\right) \cdot \nu_{k_{j+1}}\left(G \cap G_{k_{j+1}}^{*}\right) .
$$

We note that $\nu_{k_{j+1}}\left(G_{k_{j+1}}^{*}\right)=1$, therefore

$$
\nu(\Omega)=\sum_{j=0}^{+\infty} \frac{1}{2^{k_{j}}}\left(1-\left(\frac{1}{2}\right)^{k_{j+1}-k_{j}}\right) \nu_{k_{j+1}}\left(G_{k_{j+1}}^{*}\right)=\sum_{j=0}^{+\infty} \sum_{s=k_{j}+1}^{k_{j+1}} \frac{1}{2^{s}}=\sum_{s=1}^{+\infty} \frac{1}{2^{s}}=1 .
$$

Thus, $\nu$ is a probability measure defined on measurable subsets of $\Omega$. On $[0,1] \times \Omega$ we define the probability measure $P_{0}=P \otimes \nu$, which is a direct product of the probability measures $P$ and $\nu$. Now for $k \in I$ we define

$$
\begin{gathered}
F_{k}=\left\{(t, r) \in[0,1] \times \Omega: W_{N_{k}}(r, t)>A_{1} S_{N_{k}}(r) \ln ^{1 / 2} N_{k}\right\}, \\
F_{k}(r)=\left\{t \in[0,1]: W_{N_{k}}(r, t)>A_{1} S_{N_{k}}(r) \ln ^{1 / 2} N_{k}\right\},
\end{gathered}
$$

where $S_{N_{k}}^{2}(r)=\sum_{\|n\|=0}^{N_{k}}\left|a_{n}\right|^{2} r^{2 n}$ and $A_{p}$ is the constant from Lemma 1 with $\beta=1$. Using Fubini's theorem and Lemma 1 with $c_{n}=a_{n} r^{n}$ and $\beta=1$, for $k \in I$ we get

$$
P_{0}\left(F_{k}\right)=\int_{\Omega}\left(\int_{F_{k}(r)} d P\right) d \nu=\int_{\Omega} P\left(F_{k}(r)\right) d \nu \leqslant \frac{1}{N_{k}} \nu(\Omega)=\frac{1}{N_{k}} .
$$

Note that $N_{k}>\ln ^{p / 2+1} \mu_{f}\left(r^{(k)}\right) \geqslant k^{3 / 2}$. Therefore $\sum_{k \in I} P_{0}\left(F_{k}\right) \leqslant \sum_{k=1}^{+\infty} k^{-3 / 2}<+\infty$. By Borel-Cantelli's lemma the infinite quantity of the events $\left\{F_{k}: k \in I\right\}$ may occur with the zero probability. Thus,

$$
P_{0}(F)=1, \quad F=\bigcup_{s=1}^{+\infty} \bigcap_{k \geqslant s, k \in I} \overline{F_{k}} \subset[0,1] \times \Omega .
$$

Then for each point $(t, r) \in F$ there exists $k_{0}=k_{0}(t, r)$ such that for each $k \geqslant k_{0}, k \in I$ we have

$$
W_{N_{k}}(r, t) \leqslant A_{1} S_{N_{k}}(r) \ln ^{1 / 2} N_{k}
$$

Let $P_{j}$ be a probability measure defined on $\left(\Omega_{j}, \mathcal{A}_{j}\right)$, where $\mathcal{A}_{j}$ is a $\sigma$-algebra of subsets $\Omega_{j}(j \in\{1, \ldots, p\})$ and $P_{0}$ is the direct product of probability measures $P_{1}, \ldots, P_{p}$ defined on $\left(\Omega_{1} \times \ldots \times \Omega_{p}, \mathcal{A}_{1} \times \ldots \times \mathcal{A}_{p}\right)$. Here $\mathcal{A}_{1} \times \ldots \times \mathcal{A}_{p}$ is the $\sigma$-algebra, which contains all $A_{1} \times \ldots \times A_{p}$, where $A_{j} \in \mathcal{A}_{j}$. If $F \subset \mathcal{A}_{1} \times \ldots \times \mathcal{A}_{p}$ such that $P_{0}(F)=1$, then in the case when projection

$$
F_{1}=\left\{t_{1} \in \Omega_{1}:\left(\exists\left(t_{2}, \ldots, t_{p}\right) \in \Omega_{2} \times \ldots \times \Omega_{p}\right)\left[\left(t_{1}, \ldots, t_{p}\right) \in F\right]\right\}
$$

of the set $F$ on $\Omega_{1}$ is $P_{1}$-measurable we have $P_{1}\left(F_{1}\right)=1$. 
By $F_{\Omega}$ we denote the projection of $F$ on $\Omega$, i.e. $F_{\Omega}=\{r \in \Omega:(\exists t)[(t, r) \in F]\}$. Then $\nu\left(F_{\Omega}\right)=1$. Similarly, the projection of $F$ on $[0,1], F_{[0,1]}=\bigcup_{r \in \Omega} F(r)$, we obtain $P\left(F_{[0,1]}\right)=1$.

Let $F^{\wedge}(t)=\{r \in \Omega:(t, r) \in F\}$. By Fubini's theorem we have

$$
0=\int_{X}\left(1-\chi_{F}\right) d P_{0}=\int_{0}^{1}\left(\int_{\Omega}\left(1-\chi_{F^{\wedge}(t)}\right) d \nu\right) d P .
$$

Hence, $P$-almost everywhere $0=\int_{\Omega}\left(1-\chi_{F^{\wedge}(t)}\right) d \nu=1-\nu\left(F^{\wedge}(t)\right)$, i.e. $\exists F_{1} \subset F_{[0,1]}, P\left(F_{1}\right)=1$ such that for each $t \in F_{1}$ we get $\nu\left(F^{\wedge}(t)\right)=1$.

Indeed, if for some $k \in I, k=k_{j+1}$ we obtain $\nu_{k}\left(F^{\wedge}(t) \cap G_{k}^{*}\right)=q<1$, then

$$
\begin{gathered}
\nu\left(F^{\wedge}(t)\right)=\sum_{k \in I} \nu_{k}\left(F^{\wedge}(t) \cap G_{k}^{*}\right) \leqslant \sum_{s=0}^{+\infty} \frac{1}{2^{k_{s}}}\left(1-\left(\frac{1}{2}\right)^{k_{s+1}-k_{s}}\right)- \\
-(1-q) \frac{1}{2^{k_{j}}}\left(1-\left(\frac{1}{2}\right)^{k_{j+1}-k_{j}}\right)=1-(1-q) \frac{1}{2^{k_{j}}}\left(1-\left(\frac{1}{2}\right)^{k_{j+1}-k_{j}}\right)<1 .
\end{gathered}
$$

For each $t \in F_{1}$ and $k \in I$ we choose a point $r_{0}^{(k)}(t) \in G_{k}^{*}$ such that

$$
W_{N_{k}}\left(r_{0}^{(k)}(t), t\right) \geqslant \frac{3}{4} M_{k}(t), M_{k}(t) \stackrel{\text { def }}{=} \sup \left\{W_{N_{k}}(r, t): r \in G_{k}^{*}\right\}
$$

Then from $\nu_{k}\left(F^{\wedge}(t) \cap G_{k}^{*}\right)=1$ for each $k \in I$ it follows that there exists a point $r^{(k)}(t) \in$ $G_{k}^{*} \cap F^{\wedge}(t)$ such that

$$
\left|W_{N_{k}}\left(r_{0}^{(k)}(t), t\right)-W_{N_{k}}\left(r^{(k)}(t), t\right)\right|<\frac{1}{4} M_{k}(t)
$$

or

$$
\frac{3}{4} M_{k}(t) \leqslant W_{N_{k}}\left(r_{0}^{(k)}(t), t\right) \leqslant W_{N_{k}}\left(r^{(k)}(t), t\right)+\frac{1}{4} M_{k}(t) .
$$

Since $\left(t, r^{(k)}(t)\right) \in F$, from inequality 13 we obtain

$$
\frac{1}{2} M_{k}(t) \leqslant W_{N_{k}}\left(r^{(k)}(t), t\right) \leqslant A_{1} S_{N_{k}}\left(r^{(k)}(t)\right) \ln ^{1 / 2} N_{k}
$$

Now for $r^{(k)}=r^{(k)}(t)$ we get

$$
S_{N}^{2}\left(r^{(k)}\right) \leqslant \mu_{f}\left(r^{(k)}\right) \mathfrak{M}_{f}\left(r^{(k)}\right) \leqslant \mu_{f}^{2}\left(r^{(k)}\right)\left(\prod_{i=1}^{p} \ln ^{p-1} r_{i}^{(k)} \cdot \ln ^{p} \mu_{f}\left(r^{(k)}\right)\right)^{1 / 2+\delta} .
$$

Thus, for $t \in F_{1}$ and each $k \geqslant k_{0}(t), k \in I$ we obtain

$$
S_{N}\left(r^{(k)}\right) \leqslant \mu_{f}\left(r^{(k)}\right)\left(\prod_{i=1}^{p} \ln ^{p-1} r_{i}^{(k)} \cdot \ln ^{p} \mu_{f}\left(r^{(k)}\right)\right)^{1 / 4+\delta / 2} .
$$

It follows from 12 that $d_{1}\left(r^{(k)}\right) \geqslant d(r)$ for $r \in G_{k}^{*}$. Then for $t \in F_{1}, r \in F^{\wedge}(t) \cap G_{k}^{*}$, $k \in I, k \geqslant k_{0}(t)$ we get

$$
M_{f}(r, t) \leqslant \sum_{\|n\| \geqslant 2 d_{1}\left(r^{(k)}\right)}\left|a_{n}\right| r^{n}+W_{N_{k}}(r, t) \leqslant \sum_{\|n\| \geqslant 2 d(r)}\left|a_{n}\right| r^{n}+M_{k}(t) .
$$


Finally, from (11), (15), (16) for $t \in F_{1}, r \in F^{\wedge}(t) \cap G_{k}^{*}, k \in I$ and $k \geqslant k_{0}(t)$ we obtain

$$
\begin{aligned}
M_{f}\left(r^{(k)}, t\right) \leqslant & \mu_{f}\left(r^{(k)}\right)+2 A_{p} S_{N_{k}}\left(r^{(k)}\right) \ln ^{1 / 2} N_{k} \\
\leqslant & \mu_{f}\left(r^{(k)}\right)+2 A_{p} \mu_{f}\left(r^{(k)}\right)\left(\prod_{i=1}^{p} \ln ^{p-1} r_{i}^{(k)} \cdot \ln ^{p} \mu_{f}\left(r^{(k)}\right)\right)^{1 / 4+\delta / 2} \times \\
& \times\left(\left(p / 2+1+\delta_{2}\right) \ln _{2}\left(e \mu_{f}\left(r^{(k)}\right)\right)+\left(1+\delta_{2}\right) \sum_{i=1}^{p}\left(p \ln _{2} r_{i}^{(k)}+2 \ln _{3} r_{i}^{(k)}\right)\right)^{1 / 2} .
\end{aligned}
$$

Using inequality (12) we get for $t \in F_{1}, r \in F^{\wedge}(t) \cap G_{k}^{*}, k \in I$ and $k \geqslant k_{0}(t)$

$$
M_{f}(r, t) \leqslant C \mu_{f}(r)\left(\prod_{i=1}^{p} \ln ^{p-1} r_{i} \cdot \ln ^{p} \mu_{f}(r)\right)^{1 / 4+3 \delta_{2} / 4} .
$$

We choose $k_{1}>k_{0}(t)$ such that for each $r \in G_{k_{1}}^{+}$we have

$$
C \leqslant\left(\prod_{i=1}^{p} \ln ^{p-1} r_{i} \cdot \ln ^{p} \mu_{f}(r)\right)^{\delta_{2} / 4} .
$$

Using (17) and (18) we get that inequality (6) holds almost surely $\left(t \in F_{1}, P\left(F_{1}\right)=1\right)$ for each

$$
r \in\left(\bigcup_{k \in I}\left(G_{k}^{*} \cap F^{\wedge}(t)\right) \cap G_{k_{1}}^{+}\right) \backslash E^{*}=\left([1,+\infty)^{p} \cap G_{k_{1}}^{+}\right) \backslash\left(E^{*} \cup G^{*} \cup E_{p+1}\right)=[1,+\infty)^{p} \backslash E_{p+2},
$$

where

$$
E_{p+2}=E_{p+1} \cup G^{*} \cup E^{*}, \quad G^{*}=\bigcup_{k \in I}\left(G_{k}^{*} \backslash F^{\wedge}(t)\right) .
$$

It remains to observe that $\nu\left(G^{*}\right)$ defined in 13$)$ satisfies $\nu\left(G^{*}\right)=\sum_{k \in I}\left(\nu_{k}\left(G_{k}^{*}\right)-\nu_{k}\left(F^{\wedge}(t)\right)\right)=0$. Then for each $k \in I$ we obtain

$$
\begin{aligned}
\nu_{k}\left(G_{k}^{*} \backslash F^{\wedge}(t)\right) & =\frac{\operatorname{meas}_{p}\left(G_{k}^{*} \backslash F^{\wedge}(t)\right)}{\operatorname{meas}_{p}\left(G_{k}^{*}\right)}=0, \\
\operatorname{meas}_{p}\left(G_{k}^{*} \backslash F^{\wedge}(t)\right) & =\int_{G_{k}^{*} \backslash F^{\wedge}(t)} \ldots \int_{0} \frac{d r_{1} \ldots d r_{p}}{r_{1} \ldots r_{p}}=0 .
\end{aligned}
$$

\section{SOME EXAMPLES}

In this section we prove that the exponent $p / 4+\delta$ in the inequality (7) cannot be replaced by a number smaller than $p / 4$. It follows from such a statement.

Theorem 3. For $f(z)=\exp \left\{\sum_{i=1}^{p} z_{i}\right\}$ almost surely in $K(f, H)$ for $r \in E$ we have

$$
M_{f}(r, t) \geqslant \frac{1}{(8 p)^{p}} \mu_{f}(r) \ln ^{p / 4} \mu_{f}(r),
$$

where $E$ is a set of infinite asymptotically logarithmic measure and $H=\left\{e^{2 \pi i \omega_{n}}\right\},\left\{\omega_{n}\right\}$ is a sequence of independent random variables uniformly distributed on $[0,1]$.

In order to prove this theorem we need the following result.

Theorem 4 ([17]). For the entire function $g(z)=e^{z}$ almost surely in $K(g, H)$ we have

$$
\varliminf_{r \rightarrow+\infty} \frac{M_{g}(r, t)}{\mu_{g}(r) \ln ^{1 / 4} \mu_{g}(r)} \geqslant \sqrt{\frac{\pi}{8}} .
$$


Proof of Theorem 3. For the entire function $f(z)=\exp \left\{\sum_{i=1}^{p} z_{i}\right\}$ we have $\ln \mathfrak{M}_{f}(r)=\sum_{i=1}^{p} r_{i}$ and for each $\beta>0$ we get

$$
\int_{(1,+\infty)^{p}} \ldots \int_{1} \frac{d r_{1} \ldots d r_{p}}{r_{1} \ldots r_{p}\left(r_{1}+\ldots+r_{p}\right)^{\beta}}<+\infty
$$

Therefore, function $f(z)$ satisfies condition (4). From (19) we have for $r \in\left(r_{0},+\infty\right)^{p}$

$$
M_{f}(r, t)>\frac{1}{2^{p}} \mu_{f}(r) \prod_{i=1}^{p} \ln ^{1 / 4} \mu_{g}\left(r_{i}\right) .
$$

Denote $\psi(r)=\ln \mu_{g}(r)$. Note that

$$
\begin{aligned}
A_{t} & =\left\{r: r_{1}=t ; r_{i} \in\left(t_{1}, t_{2}\right)=\left(\psi^{-1}\left(\psi\left(r_{1}\right) / 2\right), \psi^{-1}\left(2 \psi\left(r_{1}\right)\right)\right)\right\} \\
& \subset\left\{r: \prod_{i=1}^{p} \psi\left(r_{i}\right) \geqslant \frac{1}{(4 p)^{p}}\left(\sum_{i=1}^{p} \psi\left(r_{i}\right)\right)^{p}\right\} .
\end{aligned}
$$

Indeed, if $r \in A_{t}$, for fixed $r_{1}$ we obtain

$$
\begin{aligned}
\prod_{i=1}^{p} \psi\left(r_{i}\right) & =\psi\left(r_{1}\right) \prod_{i=2}^{p} \psi\left(r_{i}\right)>\psi\left(r_{1}\right) \prod_{i=2}^{p} \frac{\psi\left(r_{1}\right)}{2}=\frac{\psi^{p}\left(r_{1}\right)}{2^{p-1}} \\
& =\frac{1}{2^{p-1}(2 p-1)^{p}}\left(\psi\left(r_{1}\right)+2 \psi\left(r_{1}\right)+\ldots+2 \psi\left(r_{1}\right)\right)^{p}>\frac{1}{(4 p)^{p}}\left(\sum_{i=1}^{p} \psi\left(r_{i}\right)\right)^{p}
\end{aligned}
$$

For $r \in A=\bigcup_{t=r_{0}}^{+\infty} A_{t}$ we get

$$
M_{f}(r, t)>\frac{1}{2^{p}} \mu_{f}(r) \prod_{i=1}^{p} \ln ^{1 / 4} \mu_{g}\left(r_{i}\right)>\mu_{f}(r) \frac{1}{(8 p)^{p}}\left(\sum_{i=1}^{p} \ln \mu_{g}\left(r_{i}\right)\right)^{p / 4}>\frac{1}{(8 p)^{p}} \mu_{f}(r) \ln ^{p / 4} \mu_{f}(r) .
$$

It remains to prove that the set $A$ has infinite asymptotically logarithmic measure. It is known [11] that $t<\psi^{-1}(t)<3 t / 2, t \rightarrow+\infty$. Therefore,

$$
\begin{aligned}
\operatorname{meas}_{p}(A) & =\int_{r_{0}}^{+\infty} \int_{t_{1}}^{t_{2}} \ldots \int_{t_{1}}^{t_{2}} \frac{d r_{1} \ldots d r_{p}}{r_{1} \ldots r_{p}}=\int_{r_{0}}^{+\infty}\left(\int_{t_{1}}^{t_{2}} \frac{d r_{2}}{r_{2}}\right)^{p-1} \frac{d r_{1}}{r_{1}} \\
& =\int_{r_{0}}^{+\infty}\left(\ln \psi^{-1}\left(2 \psi\left(r_{1}\right)\right)-\ln \psi^{-1}\left(\frac{\psi\left(r_{1}\right)}{2}\right)\right)^{p-1} \frac{d r_{1}}{r_{1}} \\
& >\int_{r_{0}}^{+\infty}\left(\ln \left(2 \psi\left(r_{1}\right)\right)-\ln \left(\frac{3 \psi\left(r_{1}\right)}{4}\right)\right)^{p-1} \frac{d r_{1}}{r_{1}}=\ln ^{p-1} \frac{8}{3} \cdot \int_{r_{0}}^{+\infty} \frac{d r_{1}}{r_{1}}=+\infty
\end{aligned}
$$

\section{BIBLIOGRAPHY}

1. H. Wittich. Neuere Untersuchungen über eindeutige analytische Funktionen. Springer, BerlinGöttingen-Heidelberg (1955).

2. A.A. Goldberg, B.Ja. Levin, I.V. Ostrovski. Entire and meromorphic functions // Itogi nauky i techn. VINITI 85 5-186 (1991). (in Russian.)

3. I.F. Bitlyan, A.A. Goldberg. Wiman-Valiron's theorem for entire functions of several complex variables // Vestn. Leningrad. Univ. Ser. Mat. Mech. Astr. 2:13, 27-41 (1959). (in Russian.) 
4. P.C. Fenton. Wiman-Valyron theory in two variables // Trans. Amer. Math. Soc. 347:11 4403$4412(1995)$.

5. A. Schumitzky. Wiman-Valiron theory for entire functions of several complex variables. Ph.D. Dissertation. Cornell Univ., Ithaca (1965).

6. A. Schumitzky. A probabilistic approach to the Wiman-Valiron theory for entire functions of several complex variables // Complex Variables. 13:1-2, 85-98 (1989).

7. P. Lévy. Sur la croissance de fonctions entière // Bull. Soc. Math. France 58, 29-59, 127-149 (1930).

8. P. Erdős, A. Rényi On random entire function // Zastosowania mat. 10, 47-55 (1969).

9. J. Gopala Krishna, I.H. Nagaraja Rao. Generalised inverse and probability techniques and some fundamental growth theorems in $\mathbb{C}^{k} / /$ J. Indian Math. Soc. 41, 203-219 (1977).

10. A.O. Kuryliak, O.B. Skaskiv. Wiman's type inequalities without exceptional sets for random entire functions of several variables // Matematychni Studii. 38:1, 35-50 (2012).

11. A.O. Kuryliak, L.O. Shapovalovska, O.B. Skaskiv. Wiman's type inequality for some double power series // Matematychni Studii. 39:2, 134-141 (2013).

12. O.V. Zrum, O.B. Skaskiv On Wiman's inequality for random entire functions of two variables // Matematychni Studii. 23:2, 149-160 (2005). (in Ukrainian.)

13. O.B. Skaskiv, O.V. Zrum Wiman's type inequality for entire functions of two complex variables with rapidly oscilic coefficient // Mat. Metods i Fys.-Mekh. Polya 48:4, 78-87 2005. (in Ukrainian.)

14. O.B. Skaskiv, O.V. Zrum. On inprovement of Fenton's inequality for entire functions of two complex variables // Math. Bull. Shevchenko Sci. Soc. 3, 56-68 (2006). (in Ukrainian.)

15. P.V. Filevych. Some classes of entire functions in which the Wiman-Valiron inequality can be almost certainly improved // Matematychni Studii. 6, 59-66 (1996). (in Ukrainian.)

16. P.V. Filevych. Wiman-Valiron type inequalities for entire and random entire functions of finite logarithmic order // Sib. Mat. Zhurn. 42:3, 683-694 (2003). [Siberian Math. J. 42:3, 579-586 (2003).]

17. P.V. Filevych. The Baire categories and Wiman's inequality for entire functions // Matematychni Studii. 20:2, 215-221 (2003).

Andriy O. Kuryliak,

Department of Mechanics and Mathematics,

Ivan Franko National University of L'viv,

Universytets'ka str. 1,

79000, Lviv, Ukraine

E-mail: kurylyak88@gmail.com

Oleh B. Skaskiv,

Department of Mechanics and Mathematics, Ivan Franko National University of L'viv, Universytets'ka str. 1,

79000, Lviv, Ukraine

E-mail: matstud@franko.Iviv.ua

Oleh V. Zrum,

Department of Mechanics and Mathematics, Ivan Franko National University of L'viv, Universytets'ka str. 1, 79000, Lviv, Ukraine 